\title{
INTRAOPERATIVE ULTRASONOGRAPHY IN PANCREATIC SURGERY: STAGING AND RESECTION GUIDANCE
}

\author{
O. Kolesnik*, A. Lukashenko, A. Shudrak, T. Golovko, G. Lavryk, J. Huralevych \\ National Cancer Institute, Kyiv 03025, Ukraine
}

\begin{abstract}
Background: Intraoperative ultrasound examination (IOUS) is indispensable part of modern surgical interventions in tumors of hepatopancreatobiliary zone. In this study retrospective analysis of IOUS efficiency in surgical treatment of pancreatic tumors was provided. Materials and Methods: In the period from January 2013 till November 2015 in the National Cancer Institute IOUS was applied during 76 surgical interventions: for pancreatic head tumors - in 46 (60.5\%) patients, for body/tail pancreatic tumors in $20(26.3 \%)$ patients, in $10(13.2 \%)$ patients - for periampullary zone neoplasms. In IOUS we performed primary tumor assessment (localization, degree of tumor spreading to superior mesenteric vessels, hepatoduodenal ligament vessels, additional foci occurrence in pancreas), and liver metastases detection. Surgical interventions were performed: pancreatoduodenectomy in 52 (68.4\%) patients, radical antegrade modal pancreatosplenectomy - in 14 (18.4\%) patients. Results: IOUS allowed determining additional tumor foci in pancreas in $2(2.6 \%)$ patients, in $8(\mathbf{1 0 . 5 \% )}$ observations tumor invasion into portal or superior mesenteric vein was determined. In 21 (27.6\%) patient additional hepatic neoplasms were detected (in half of cases $-\mathbf{1 1 . 8 \%}$, metastases). These findings resulted in change of surgical intervention extent in $23(30.3 \%)$ patients: expansion to combined resections in $14(18.4 \%)$ patients, reduction to symptomatic operations in $5(6.6 \%)$ cases, organ-preserving operations were performed in $4(5.3 \%)$ patients. Variant anatomy of hepatic arterial blood supply was determined in 41 (53.9\%) patients that necessitated performance correction of resection stages for preservation of adequate hepatic blood supply. Conclusions: IOUS is a highly-precise diagnostic method substantively influencing operation course in pancreatic tumors, enabling surgeon to provide adequate staging and permanent correction of operation course. Key Words: intraoperative ultrasound examination, pancreatic tumors surgery.
\end{abstract}

Precise preoperative staging of tumor process is a basic principle for choice of optimal extent for surgical intervention. Intraoperative ultrasound examination (IOUS) in a number of situations is superior to spiral computer tomography (CT) and magnetic resonance tomography (MRI) in terms of sensitivity and specificity, at present it is essentials for surgical interventions for hepatopancreatobiliary tumors [1]. Enhancement of radiologic methods precision remains actual problem, especially for diagnostics of pancreatic tumor spreading, where currently the term "borderline resectable tumors" remains generally accepted [2].

IOUS was applied for the first time in 1960 s to localize kidney stones. At early stages the novel method was rather skeptically met by surgeons, and only closer to 1970 s IOUS began to gain increasing popularity, which was facilitated by equipment improvement, introduction of new, higher frequencies for examination, and imaging quality increase. Nevertheless for the first time IOUS in pancreatic tumors was applied only in 1980 [3, 4].

According to a number of authors in operative interventions in hepatopancreatobiliary zone IOUS could alter surgical intervention plan in $4.5-40 \%$ of cases [5, $6]$. IOUS of pancreas allows precise main pancreatic

\section{Submitted:}

${ }^{\star}$ Correspondence: E-mail: mail.onco@gmail.com

Abbreviations used: CT - computer tomography; IOUS - intraoperative ultrasound; ISGPF - International Study Group on Pancreatic Fistula; MRCP - magnetic resonance cholangiopancreatography; MRI - magnetic resonance tomography; NET - neuroendocrine tumor; PD - pancreatoduodenectomy; PET-CT - positron emission tomography - computed tomography; RAMPS - radical antegrade modular pancreatosplenectomy. duct localization in glandular tissue, determines its relation towards tumor, clarifies occurrence and degree of canal blockage with tumor, and possible retention cysts occurrence. Application of Doppler IOUS regimen extends possibilities for intraoperative localization of vessels of hepatoduodenal ligament and mesentery, allows determining their variant anatomy and also occurrence and degree of tumor invasion into main visceral vessels. At liver metastases detection IOUS significantly exceeds CT or MRI in sensitivity parameter, reaching $94-96 \%$ even at small foci with 3-5 mm dimensions [7].

To determine effectiveness of IOUS in pancreatic cancer surgery, we analyzed our own experience of this method application.

\section{MATERIALS AND METHODS}

In the period from January 2013 till November 2015 in the clinics of the National Cancer Institute IOUS procedure was applied for 76 surgical interventions for pancreatic tumor. Average patients' age was $62.5 \pm 3.6$, males to females ratio was $33 / 43$, respectively. In $46(60.5 \%)$ patients the primary tumor was localized in pancreatic head, in 20 patients $(26.3 \%)$ - in pancreatic body/tail, $13.2 \%$ of cases corresponded to periampullary zone tumors (major duodenal papilla, terminal choledoch units, and duodenum). This study was approved by local ethical committee. Informed consent was obtained from each patient. Patients' characteristics are presented in Table 1. Whole malignancies in presented series were confirmed histologically.

All the patients received standard preoperative examination that mandatory included multi-slice abdominal CT with intravenous contrast enhancement, 
followed by three-dimensional vascular reconstruction. At surgical interventions planning CT data that were received no later than 4 weeks prior to surgery date were used. CT assessment was carried out by radiologists with broad experience in diagnostics of hepatopancreatobiliary tumors. Three patients apart from CT additionally received 18-fluorodeoxyglucose positron emission computer tomography (PET-CT), 21 (26.4\%) patients additionally received MRI. 34 (44.7\%) patients received percutaneous transhepatic biliary drainage for biliary decompression for tumors complicated by obstructive jaundice at preoperative stage.

Table 1. Patients characteristics

\begin{tabular}{lcc}
\hline \multicolumn{1}{c}{ Characteristics } & $\mathrm{n}$ & $\%$ \\
\hline General patients' number in the study & 76 & \\
Average age, years & $62.5 \pm 3.6$ & \\
Ratio males/females & $33 / 43$ & \\
Tumor localization & 46 & 60.5 \\
Pancreatic head & 10 & 13.2 \\
Periampullary zone: & 5 & 6.6 \\
- major duodenal papilla & 3 & 3.9 \\
- distal choledochus & 2 & 2.6 \\
- duodenum & 20 & 26.3 \\
Pancreatic body/tail & & \\
Additional examination methods & 21 & 27.6 \\
MRI, magnetic resonance cholangiopancreatography & & \\
(MRCP) & 3 & 3.9 \\
PET-CT & & \\
Method of biliary decompression at mechanical jaundice & 34 & 44.7 \\
Percutaneous transhepatic biliary drainage & 5 & 6.6 \\
Biliary stenting & &
\end{tabular}

IOUS procedure. IOUS was performed with devise FlexFocus 400 and UltraView 800 (BK, Denmark) with application of convex, biplane and laparoscopic transducers. Transducers technical characteristics are presented in Table 2.

Study protocol was standardized for all the patients. Major IOUS steps included clarification of tumor localization in pancreas, assessment of its texture, relationships with vessels of hepatoduodenal ligament, mesentery, and celiac artery. Additional IOUS target was assessment of healthy gland parenchyma, additional tumor foci presence, major pancreatic duct localization, its dilatation degree, and cysts occurrence in pancreatic tissue. After pancreas examination liver IOUS was carried out for metastasis detection and differential diagnostics with benign liver neoplasms.

IOUS technique. After surgical site preparation IOUS through gastric wall was started, using stomach as acoustic window; direct pancreas examination was performed after gastrocolic ligament mobilization. As main acoustic markers the portal veins were used, starting from confluence and further along splenic and superior mesenteric veins. Additional acoustic markers were superior mesenteric artery, gastroduodenal artery, and celiac artery. After regional lymph nodes assessment (lymph nodes of hepatoduodenal ligament, aortocaval group, etc.), choledoch and Wirsung duct were examined.

At liver IOUS for better visualization of frontal organ surface falciform ligament transection was performed. Further at right lobe IOUS the liver was slightly translocated by round ligament abduction to the left. Optimal image obtaining was achieved by liver capsule irrigation with $20 \mathrm{ml}$ of saline. First point of orientation was entry of median hepatic vein, exploration was provided starting from "initial triangle" formed by three hepatic veins toward periphery using inferior vena cava as additional orientation. Segmental portal branches that centrifugally directed from portal fissure toward periphery were explored segment by segment, starting from entry; at that topographical marking was performed according to Couinaud's classification. Important step was determination of Glissonian pedicle to every liver segment. Detection of all auxiliary structures not related to Glisson's entity further was explored in more detail by duplex regimen application. At parenchyma assessment main consideration was given to masses with texture different from normal liver parenchyma.

Table 2. Technical characteristics of transducers

\begin{tabular}{lccc}
\hline $\begin{array}{c}\text { Characte- } \\
\text { ristics }\end{array}$ & $\begin{array}{c}\text { Frequency, } \\
\mathrm{MHz}\end{array}$ & $\begin{array}{c}\text { Focal length, } \\
\mathrm{mm}\end{array}$ & $\begin{array}{c}\text { Contact area, } \\
\mathrm{mm}\end{array}$ \\
\hline Linear & $12-5$ & $2-55$ & $50 \times 4$ \\
Convex & $6-2$ & $12-200$ & $65.5 \times 13$ \\
Biplane & $10-3.75$ & $5-95$ & $5 \times 30 \times 2$ \\
Laparosco- & $10-4.3$ & $5-95$ & $5 \times 30$ \\
pic-4-Way & & & \\
\hline
\end{tabular}

IOUS was completed with determination of anatomic variant of common hepatic artery, proper hepatic artery and lobular hepatic arteries, and also the variant of portal vein confluence. In case of pancreatic tumor contact with hepatic artery, superior mesenteric artery of celiac artery, more detailed assessment of blood flow changes in these vessels and also of blood flow in liver peripheral vessels was conducted for confirmation of true arterial invasion.

In case of tumor contact with the wall of portal vein or dorsal mesenteric vein we determined the type of venous invasion according to Nakao classification [8], followed by choice of optimal tactics for portal resection/reconstruction.

Before the end of surgical intervention we performed ultrasound duplex assessment of liver arterial blood flow to exclude possible thrombosis resulting from surgical manipulations (lymph node dissections, angioplasty, etc). During implementation of portal resection/reconstruction we assessed portal blood flow both in resection zone, and in peripheral intrahepatic portal branches.

\section{RESULTS}

Average duration of the IOUS procedure was $17 \pm 5$ min. In 10 cases IOUS were conducted for periampullary tumors (adenocarcinoma of major duodenal papilla, terminal choledoch, and duodenum). In 66 cases of primary pancreatic tumors, the ductal adenocarcinoma was in $68.2 \%$ (45 patients). These tumors in $80 \%$ were hypoechoic comparing to surrounding pancreatic tissue, and were hyperechoic in $15.6 \%$ of cases. Neuroendocrine tumors (NET) comprised $18.2 \%$ (12 patients); at that $91.7 \%$ of tumors were hyperechoic. In other pancreatic tumors all neoplasms were hyperechoic (Table 3). 
Table 3. IOUS tumor characteristic based on morphologic characteristics

\begin{tabular}{lccc}
\hline \multicolumn{1}{c}{ Tumor } & $\begin{array}{c}\text { Hypoechoic } \\
\text { tumors, } \mathrm{n}(\%)\end{array}$ & $\begin{array}{c}\text { Hyperechoic } \\
\text { tumors } \mathrm{n}(\%)\end{array}$ & Total, $\mathrm{n}$ \\
\hline Pancreatic ductal adenocarcinoma & $38(84.4)$ & $7(15.6)$ & 45 \\
NET & $11(91.7)$ & $1(8.3)$ & 12 \\
Acinic cell adenocarcinoma & $2(66.6)$ & $1(33.3)$ & 3 \\
Solid pseudopapillary tumor & $5(100.0)$ & - & 5 \\
Pancreatic sarcoma & $1(100.0)$ & - & 1 \\
Periampullary zone adenocarcinoma & $10(100.0)$ & - & 10 \\
Total & & & 76 \\
\hline
\end{tabular}

In 21 patients (27.6\%) IOUS discovered additional neoplasms in liver. Metastatic liver affection was diagnosed in 9 patients (11.8\%); from 6 of them (7.9\%) we revealed ductal adenocarcinoma metastases, in 3 patients (3.9\%) metastases of NET. These findings resulted in extension of surgical procedure in 4 patients $(5.3 \%)$, and in decrease of surgical procedure in $5 \mathrm{pa}-$ tients (6.6\%). Benign liver neoplasms were diagnosed in 12 cases (15.8\%), including 5 cases that previously were assessed as "suspicious" for metastatic liver affection by CT and MRI. In 4 cases with Doppler IOUS the differential diagnostics of hepatic cysts with tumor metastases was conducted. More detailed description of obtained results is presented in Table 4.

During IOUS additional pancreatic tumor lesions were detected in 2 patients (2.6\%) that led to extension of resection in both cases. In two other cases at palpatory examination we failed to detect tumor foci that were previously determined by CT. In this situation IOUS allowed realizing meticulous intraoperative navigation resulting in clear surgical resection margin (R0) (Fig. 1).

During IOUS variant anatomy of right branch of proper hepatic artery was detected in $36.8 \%$ cases, of left branch of proper hepatic artery - in $17.1 \%$ cases that in all the patients necessitated resection performance correction for preservation of adequate hepatic blood supply.

Table 4. Data received at IOUS

\begin{tabular}{|c|c|c|}
\hline Data received at IOUS & $\mathrm{n}$ & $\%$ \\
\hline The following lesions were additionally detected in liver: & 21 & 27.6 \\
\hline - metastases of pancreatic ductal adenocarcinoma & 6 & 7.9 \\
\hline - metastases of neuroendocrine pancreatic tumor & 3 & 3.9 \\
\hline - hepatic cysts & 4 & 5.3 \\
\hline - hepatic hemangiomas & 8 & 10.5 \\
\hline $\begin{array}{l}\text { The following lesions were additionally detected in pan- } \\
\text { creas }\end{array}$ & 2 & 2.6 \\
\hline Tumor invasion into portal system veins: & 8 & 10.5 \\
\hline lakao & 4 & 5.3 \\
\hline - type B b & 2 & 2.6 \\
\hline - typ & 3 & 3.9 \\
\hline langed after IOUS & 23 & 30.3 \\
\hline oanded: & 14 & 18.4 \\
\hline tion +1 & 4 & 5.3 \\
\hline createctomy & 1 & 1.3 \\
\hline $\begin{array}{l}\text { Whipple procedure } \rightarrow \text { combined Whipple procedure with } \\
\text { resection of pancreatic head/tail }\end{array}$ & 1 & 1.3 \\
\hline $\begin{array}{l}\text { Whipple procedure } \rightarrow \text { combined Whipple procedure with } \\
\text { resection of portal/upper mesentery veins }\end{array}$ & 6 & 7.9 \\
\hline $\begin{array}{l}\text { RAMPS } \rightarrow \text { combined RAMPS with resection of portal/up- } \\
\text { per mesentery }\end{array}$ & 2 & 2.6 \\
\hline ention was & 9 & 11.8 \\
\hline erving distal pancreatic resection & 4 & 5.3 \\
\hline tic surgery & 5 & 6.6 \\
\hline $\mathrm{n}$ the lis & 41 & 53.9 \\
\hline of righ & $2 \varepsilon$ & 36.8 \\
\hline - arterial va & 13 & 17.1 \\
\hline
\end{tabular}

Note: RAMPS - radical antegrade modular pancreatosplenectomy.

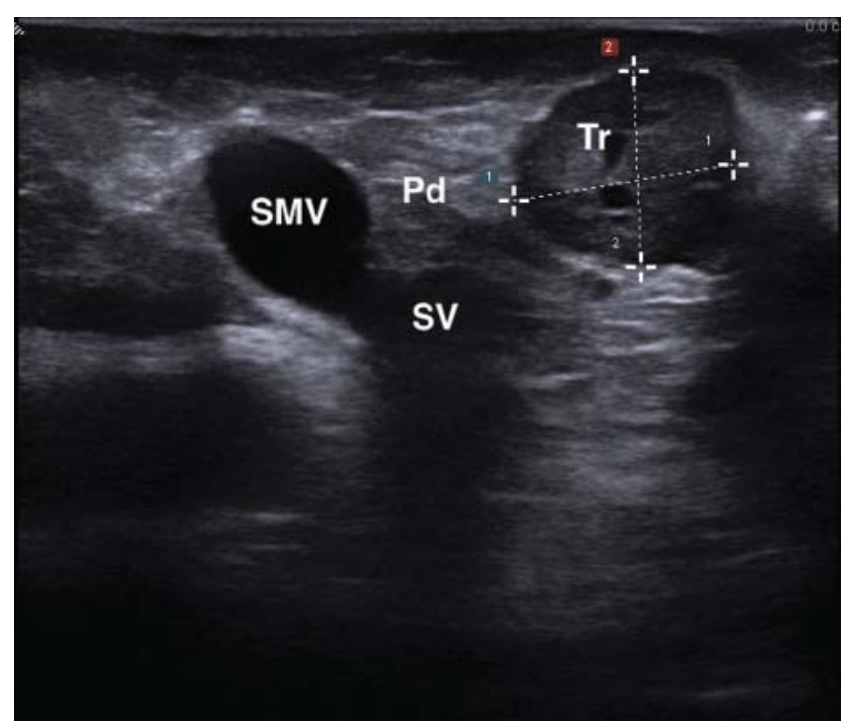

Fig. 1. IOUS navigation in pancreatic body tumor with maximal diameter $1.1 \mathrm{~cm}$ that is not detectable during palpation; SMV superior mesenteric vein; SV - splenic vein, $\mathrm{Tr}$ - tumor

In 8 patients IOUS revealed tumor invasion into portal or superior mesenteric vein that led to expanding of surgical resection up to combined one with resection/reconstruction of portal system veins.

Characteristics of surgical treatment and postoperative complications. 52 (68.4\%) patients with localization of primary tumor in pancreatic head or in periampullary zone were subjected to pancreatoduodenectomy (PD); for tumors of distal departments of pancreas in 14 patients radical antegrade modular pancreatosplenectomy (RAMPS) was performed (Table 5). In 4 cases of solid pseudopapillary tumor, and also in 2 cases of NET, localized in pancreatic body/tail, IOUS helped to confirm the absence of tumor encasement of splenic artery/vein; therefore spleen-preserving distal pancreatic resections were performed.

In two patients at PD planning additional foci in pancreatic head/tail were identified with IOUS. In one case intervention was expanded to total pancreatectomy at multifocal growth form of pancreatic cancer. In another case at confirmation of 3 NET foci in pancreatic head with dimensions from 3 to $7 \mathrm{~cm}$ and presence of 2 additional foci in pancreatic isthmus and tail - of 10 and $5 \mathrm{~mm}$, respectively, resection extent was expanded to combined PD with resection of pancreatic body and resection of portion of pancreatic tail with spleen preservation (Fig. 2).

Table 5. Surgical procedure, postoperative complications rate

\begin{tabular}{lcc}
\multicolumn{1}{c}{ Surgical procedure, postoperative complications rate } & $\mathrm{n}$ & $\%$ \\
\hline Radical resection: & 71 & 93.4 \\
- whipple procedure & 52 & 68.4 \\
- RAMPS & 14 & 18.4 \\
- total pancreatectomy & 1 & 1.3 \\
- spleen-preserving distal pancreatectomy & 4 & 5.3 \\
Symptomatic operations & 5 & 6.6 \\
Multivisceral resections: & & \\
- left-sided nephrectomy & 3 & 3.9 \\
- diaphragm resection & 2 & 2.6 \\
- colectomy & 5 & 6.6 \\
- right-sided hemihepatectomy & 1 & 1.3 \\
- proximal gastric resection & 1 & 1.3 \\
- atypical hepatic resection & 3 & 3.9 \\
Resection of portal system veins: & 8 & 10.5 \\
- linear vein resection & 5 & 6.6 \\
- circular vein resection & 3 & 3.9
\end{tabular}




\begin{tabular}{lcc}
\hline \multicolumn{1}{c}{ Surgical procedure, postoperative complications rate } & $\mathrm{n}$ & $\%$ \\
\hline Morphological examination data (71 radical resection): & & \\
- R0 resection & 64 & 90.1 \\
- R1 resection & 7 & 9.9 \\
True invasion into vascular wall (from 8 cases of portal re- & 7 & 87.5 \\
section): & 4 & 57.1 \\
- invasion into vascular adventitia & 3 & 42.9 \\
- invasion into proper muscular layer & 29 & 38.2 \\
Postoperative complications & 26 & 37.1 \\
Pancreatic fistula (70 cases of pancreatic resection), from & & \\
them: & 20 & 76.9 \\
• type A & 4 & 15.4 \\
- type B & 2 & 7.7 \\
- type C & 11 & 14.7 \\
Acute pancreatitis & 15 & 19.7 \\
Delayed gastric empting & 10 & 13.2 \\
Intraabdominal fluid collection & 5 & 6.6 \\
Surgical wound infection & 7 & 9.2 \\
Need for postoperative additional US-guided drainage & 1 & 1.3 \\
Need for relapatotomy & 1 & 1.3 \\
Postoperative mortality & & \\
\hline
\end{tabular}

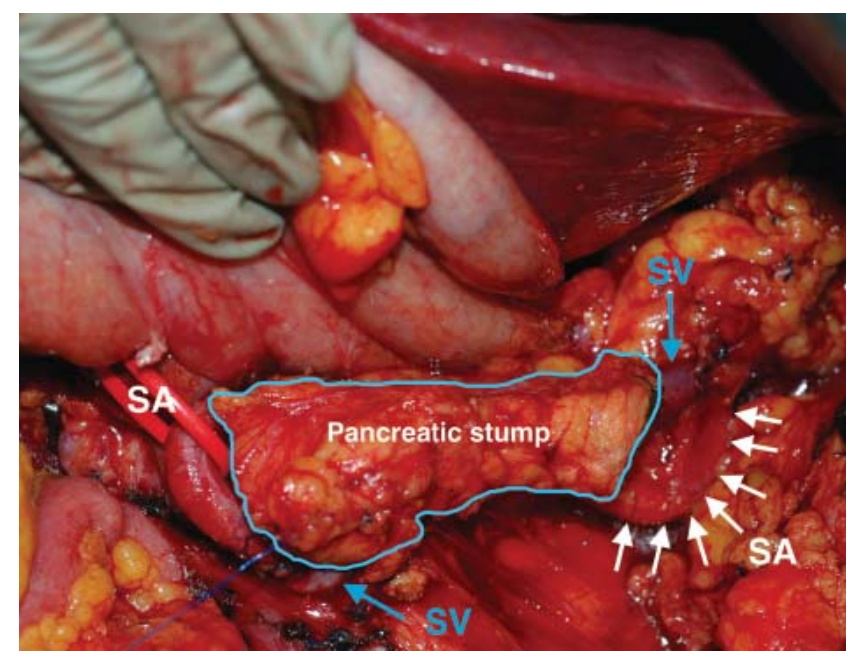

Fig. 2. Intraoperative photo: Whipple procedure with pancreatic

tail resection and preservation of splenic vein and splenic artery,

SA — splenic artery, SV — splenic vein

In the presence of IOUS data about involvement

of portal or superior mesenteric vein into tumor pro-

cess in case of tumor affection of less than 180 de-

grees of vessel circumference the linear resection

(Fig. 3, a, b) was performed of portal or superior

mesenteric vein (Fig. 4, a) in 5 patients (6.6\%), and

3 patients with larger affection type - B-C by Nakao

classification - were subjected to portomesenterial

anastomosis "end-to-end" (Fig. 4, b, c, d).
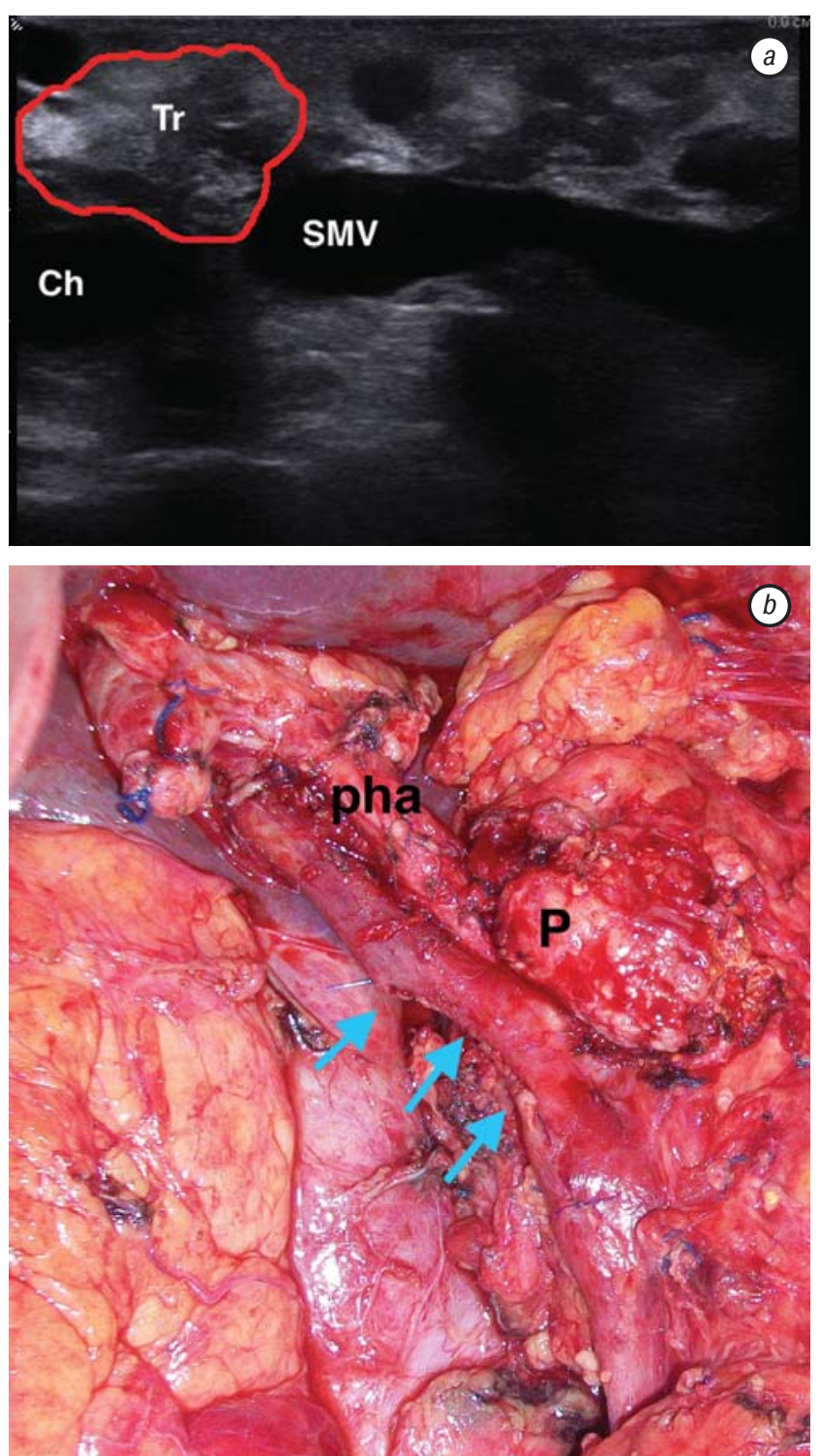

Fig. 3. Tumor is located between choledoch and superior mesenteric vein with moderate deformation of the vascular wall (type A according to Nakao classification); SMV - superior mesenteric vein, $\mathrm{Ch}$ - choledoch, $\mathrm{Tr}$ - tumor (a). Linear resection of portal and superior mesenteric veins (intraoperative picture of the case; arrows indicate vascular suture line); $\mathrm{P}$ - pancreatic stump, pha - proper hepatic artery $(b)$

At metastases in liver in 3 cases (in 2 patients with pancreatic NET and in one patient with ductal adenocarcinoma) atypical liver resections were performed; in one case it was multivisceral resection: RAMPS with right-sided hemihepatectomy at metastases of metastatic high-differentiated NET. In other three cases after confirmation of adenocarcinoma metastases into liver surgical intervention was reduced to palliation.

According to the data of final morphological examinations $\mathrm{R} 0$ resection was achieved in 64 (90.1\%) from 71 patients that were subjected to radical surgical interventions. At resection of portal system veins true invasion was confirmed in 7 of 8 cases, at that in 4 observations the tumor invaded into vessel adventitia, and in 3 cases - into muscular layer (Fig. 4, e).

Major complication was pancreatic fistula - in 37\% of cases, predominantly of low and moderate severity grade (type A-B according to International Study Group on Pancreatic Fistula — ISGPF) [9]; only in 9.2\% 

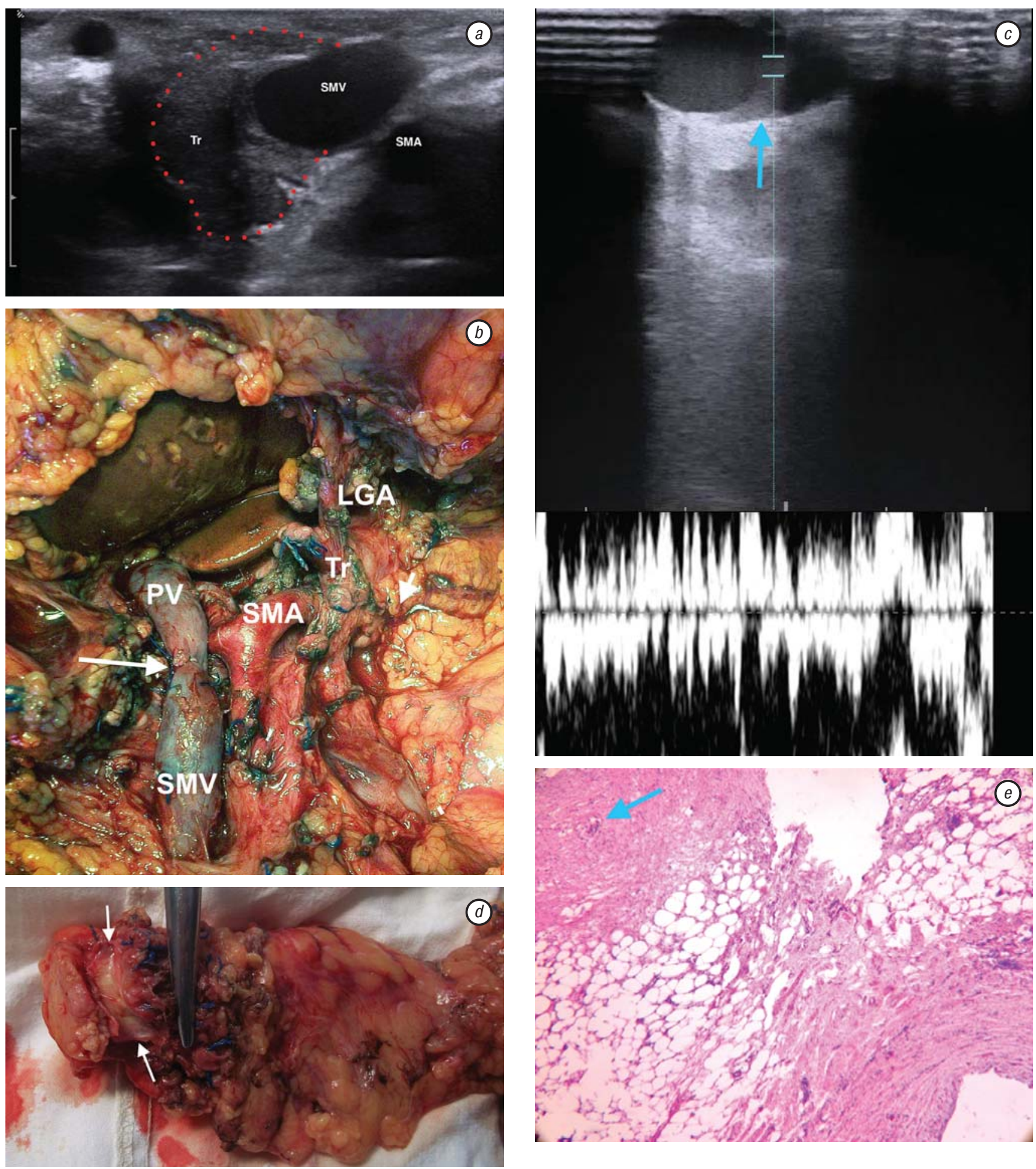

Fig. 4. Advanced cancer of pancreatic body, involvement of the superior mesenteric vein (SMV); SMA - superior mesenteric artery, $\mathrm{Tr}$ - tumor (a). RAMPS was performed with circular resection of superior mesenteric vein; vascular suture line is indicated with arrow (intraoperative picture of the case): PV - portal vein, SMV - superior mesenteric vein, SMA — superior mesenteric artery, $\mathrm{Tr}$ - coeliac trunk, LGA - left gastric artery $(b)$. Zone of circular portomesenterial anastomosis (indicated with arrow). IOUS control after circular resection/reconstruction. Estimation of portal blood flow sufficiency $(c)$. Macroscopic specimen - circular mesenteric vein resection in RAMPS (arrows indicate resection margins of superior mesenteric vein; $d$ ). Pathomorphological examination confirmed true invasion into muscular layer of superior mesenteric vein wall (arrow indicates tumor cells; e)

the need for additional US-guided drainage arose. Total postoperative lethality was $1.3 \%$

\section{DISCUSSION}

Preoperative staging at pancreatic cancer remains actual despite of emergence of novel diagnostics methods; explorative interventions continue to make significant share (20-57\%). Major difficulties are related to determination of local resectability [10] and distant metastases detection [11] that supports the need to continue studies in this area. Experience in diagnostics of neoplasms of hepatopancreatobiliar system is very important because it reliably increases accuracy of CT and MRI examinations in highly specialized centers [12].

Determination of the extent of pancreatic neoplasm encasement of superior mesenteric vessels and also of hepatoduodenal ligament vessels - is one of the key moments in tumor resectability evaluation. Modern 
procedures CT and MRI with application of technology of three-dimensional reconstruction have high sensitivity and specificity for vascular invasion diagnostics [13]. At the same time IOUS demonstrated superiority over CT in the study of Soyer et al.; tumor encasement of vessels was additionally detected in $15 \%$ of patients with absence of invasion signs at CT [14]; in our study this index comprised $10.5 \%$. At further morphological examination true invasions were confirmed in $87.5 \%$ of cases that was somewhat higher than figures reported in previous studies (26-85\%) [13, 14]. Thereby IOUS is effective method that allows determining the presence of true tumor invasions into vessels. In case of tumor encasement of portal system veins IOUS allowed classification of portal invasion type and choosing optimal ways for portal resection/reconstruction.

In 41 (53.9\%) patients in our study the presence of hepatic artery variant anatomy required special attention at pancreas mobilization. The slightest trauma of variantly located both right hepatic artery at PD and left hepatic artery at distal pancreatic resections subsequently leads to arterial thrombosis with development of necrosis of appropriate liver portion. Modern IOUS enables intraoperative Doppler control of satisfactory arterial and portal blood flow in great and peripheral hepatic vessels after completion of hepatoduodenal ligament lymph dissection, and also in cases of resection/reconstruction of portal system veins [12].

At metastatic liver affection IOUS supersedes CT, MRI in terms of sensitivity and specificity by $5-30 \%$ [10, 11, $15,16]$. Whereas CT/MRI data are limited in determination of foci less than $2 \mathrm{~cm}$, IOUS augments visualization threshold at solid foci up to 3-5 mm, at cystic foci up to 1-3 mm [6]. Metastases of pancreatic cancer in the majority of cases were hypoechoic $(84.4 \%$ of cases in our study) that required differential diagnostics with hemangiomas, hepatic adenomas, cirrhotic nodules and other benign liver tumors. Benign hepatic formations do not engage central vascular structures; they only alter their contour, while malignant tumors trend to invade vascular lumen with partial occlusion and also with thrombosis. Application of hepatic IOUS allows for precise localization of affected segment according to Coinaud classification, and also to determine tumor topography with respect to hepatic veins and portal structures.

In this study additional hepatic neoplasms were determined in $27.6 \%$, at that metastatic liver affection comprised about half of cases (11.8\%). The fact that these neoplasms with dimensions from 0.5 to $1.5 \mathrm{~cm}$ were not previously visualized by preoperative $\mathrm{CT}, \mathrm{MRI}$ and $\mathrm{PET}-\mathrm{CT}$, and also by palpation deserves special attention. IOUS sensitivity at pancreatic NET varies from 80 to $95 \%$, major limitation is focus dimensions [17]. Study of Kauhanen et al. demonstrated that PET-CT has higher sensitivity than CT and MRI for diagnostics of distal NET metastases [18]. In one patient with NET in our study IOUS allowed determining additional foci in pancreas with dimensions $1 \mathrm{~cm}$ and $0.5 \mathrm{~mm}$ (these lumps by CT were regarded as cysts).
At pancreatic cancer important prognostic factor is resection margin status. In case of small tumors that can hardly be discerned by palpation the control of adequate (R0) margin may be complicated. In our study IOUS appeared to be the indispensable method of intraoperative navigation for tumors less than $1 \mathrm{~cm}$ in 2 cases. Additional IOUS data led to alteration of extent of surgery in one third of patients (30.1\%): extent of resection was expanded to combined resection in $18.4 \%$ of cases, and it was reduced to symptomatic in $6.6 \%$. In $5.6 \%$ of cases IOUS data allowed to perform organ-saving operations. Surgical intervention correction according to variant vascular anatomy was required in $53.9 \%$ of cases.

According to the data received in our study IOUS proved to be the method that is convenient to use, and which only insignificantly extends operation period. Additional advantages of this method over CT and MRI were dynamic image obtaining with possibility of correction and new data acquisition at any moment of operation.

\section{CONCLUSIONS}

At pancreatic tumors IOUS has higher sensitivity and specificity than preoperative transabdominal US examination, CT, MRI, and also it precedes capacities of operational visual and palpatory diagnostics.

IOUS allows carrying out intraoperative assessment of variant vessels anatomy with appropriate correction of operation strategy, and also Doppler control estimation of vascular perfusion before operation completion.

At present IOUS is indispensable part of all diagnostic and treatment complex for pancreatic tumors. This is a safe method which insignificantly extends time of operative intervention.

\section{REFERENCES}

1. Cirimbei S, Puscu C, Lucenco L, Bratucu E. The role of intraoperative ultrasound in establishing the surgical strategy regarding hepato-bilio-pancreatic pathology. Chirurgia 2013; 108: $643-51$.

2. Katz MH, Marsh R, Herman JM, et al. Borderline resectable pancreatic cancer: need for standardization and methods for optimal clinical trial design. Ann Surg Oncol 2013; 20: 2787-95.

3. Machi J, Sigel B, Zaren HA, et al. Operative ultrasonography during hepatobiliary and pancreatic surgery. World J Surg 1993; 17: 640-5.

4. Botea F, Sarbu V, Dima S, et al. The role of intraoperative ultrasound in the diagnosis and treatment of hydatid liver disease. Chirurgia 2006; 101: 593-8.

5. Kane RA, Hughes LA, Cua EJ, et al. The impact of intraoperative ultrasonography on surgery for liver neoplasms. J Ultrasound Med 1994; 13: 1-6.

6. Luck AJ, Maddern GJ. Intraoperative abdominal ultrasonography. Br J Surg 1999; 86: 5-16.

7. Ni Mhuircheartaigh JM, Sun MR, Callery MP, et al. Pancreatic surgery: a multidisciplinary assessment of the value of intraoperative US. Radiology 2013; 266: 945-55.

8. Nakao A, Kanzaki A, Fujii T, et al. Correlation between radiographic classification and pathological grade of portal vein wall invasion in pancreatic head cancer. Ann Surg 2012; 255: $103-8$. 
9. Bassi C, Dervenis C, Butturini G, et al. Postoperative pancreatic fistula: an international study group (ISGPF) definition. Surgery 2005; 138: 8-13.

10. Takigawa Y, Sugawara Y, Yamamoto J, et al. New lesions detected by intraoperative ultrasound during liver resection for hepatocellular carcinoma. Ultrasound Med Biol 2001; 27: 151-6.

11. Torzilli G, Makuuchi M. Intraoperative ultrasonography in liver cancer. Surg Oncol Clin N Am 2003; 12: 91-103.

12. Barabino M, Santambrogio R, Pisani Ceretti A, et al. Is there still a role for laparoscopy combined with laparoscopic ultrasonography in the staging of pancreatic cancer? Surg Endosc 2011; 25: 160-5.

13. Solomon MJ, Stephen MS, Gallinger S, White GH. Does intraoperative hepatic ultrasonography change surgical decision making during liver resection? Am J Surg 1994; 168: $307-10$.

14. Soyer P, Levesque M, Elias D, et al. Detection of liver metastases from colorectal cancer: comparison of intraopera- tive US and CT during arterial portography. Radiology 1992; 183: $541-4$.

15. Cerwenka H, Raith J, Bacher H, et al. Is intraoperative ultrasonography during partial hepatectomy still necessary in the age of magnetic resonance imaging? Hepatogastroenterology 2003; 50: 1539-41.

16. Zhang K, Kokudo N, Hasegawa K, et al. Detection of new tumors by intraoperative ultrasonography during repeated hepatic resections for hepatocellular carcinoma. Arch Surg 2007; 142: 1170-5.

17. Virgolini I, Traub-Weidinger T, Decristoforo C. Nuclear medicine in the detection and management of pancreatic islet-cell tumours. Best Pract Res Clin Endocrinol Metab 2005; 19: 213-27.

18. Kauhanen SP, Komar G, Seppanen MP, et al. A prospective diagnostic accuracy study of ${ }^{18} \mathrm{~F}$-fluorodeoxyglucose positron emission tomography/computed tomography, multidetector row computed tomography, and magnetic resonance imaging in primary diagnosis and staging of pancreatic cancer. Ann Surg 2009; 250: 957-63. 